\title{
TRIAGE: Applying Context to Improve Timely Delivery of Critical Data in Mobile Ad Hoc Networks for Disaster Response
}

\author{
Faisal Luqman \\ Electrical and Computer Engineering \\ Carnegie Mellon University, Silicon Valley \\ Moffett Field, USA \\ faisal.luqman@sv.cmu.edu
}

\begin{abstract}
In disaster response, critical data needs to be delivered quickly so responders can address high priority issues as soon as they occur. Mobile ad hoc networks are fundamental in these efforts as the existing telecommunications infrastructure is often rendered inaccessible in large-scale disasters. Due to the inherent low bandwidth in mobile ad hoc networks, an explosion of data may cause congestion. We present TRIAGE, a framework that leverages sender context information to ensure the timely delivery of critical data.
\end{abstract}

Keywords-collaboration; disaster response; context-aware; ad hoc networks; mobility

\section{INTRODUCTION}

In mobile environments, a user's context changes rapidly and is typically accompanied by an increased volume of realtime data. In large-scale disasters, this explosion of sensor data, images, sound and messages can potentially overwhelm both the underlying network and the response coordinators. Furthermore, this data increase may cause congestion in ad hoc networks, where traffic patterns are not known a priori. Congestion is further aggravated by low bandwidth in disaster response environments. Thus, the question we seek to address is: How do we ensure the timely delivery of critical data packets in a congested mobile ad hoc network?

Though TCP guarantees packet delivery in critical environments, it does not perform well in wireless networks due to its inability to differentiate between link failures and congestion loss. TCP invokes congestion control mechanisms upon packet loss, which introduces delay. In disaster response, it is desirable that critical data reaches the recipient as quickly as possible. Increased delays may cause slow response times, poor performance and loss of life.

Given the bandwidth constraints in mobile ad hoc networks, it is not possible for all packets to be sent with minimal delay. TRIAGE represents our initial approach to developing a framework that exploits context information to prioritize critical packets. TRIAGE utilizes proactive buffer monitoring, hop-by-hop and centralized congestion avoidance, and sender rate adaption based on context information and network feedback.

Reliable packet delivery and congestion avoidance in MANETs has been an active area of research. We leverage techniques from previous work in our design. Sundaresan et al. describe ATP [1], a transport protocol that dynamically changes the sender's transmission rate based on intermediate node feedback. Hull et al. describe Fusion [2], which mitigates congestion in wireless sensor networks using a combination of hop-by-hop back pressure, rate limiting and MAC layer prioritization. Zorzi et al. present GeRaF [3], a forwarding technique based on geographical node location and contention

The rest of this paper is organized as follows. We describe our framework and system design in Section II, and our planned experiments and evaluation metrics in Section III. In Section IV, we discuss challenges and future work.

\section{TRIAGE FRAMEWORK}

\section{A. Network Model and Assumptions}

We assume a multi-hop mobile ad hoc network of approximately 200 nodes, consisting of smartphones that can run IEEE $802.11 \mathrm{~b}$ in wireless ad hoc mode, such as the Motorola Droid and Google Nexus One. We build on TCP as the transport protocol and AODV as the routing protocol. We assume bursty network traffic with nodes sending periodic and event correlated data. Finally, we assume a high fan-in network topology, typical in hastily formed networks.

\section{B. System Overview}

Figure 1 provides an overview of the system and describes the information flow.

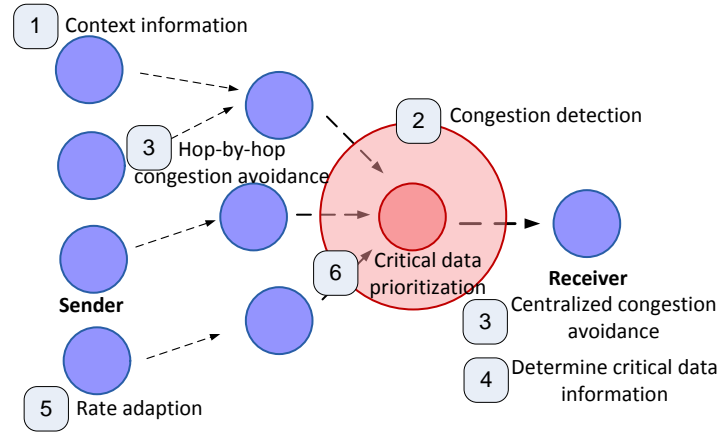

Figure 1. Information flow.

First, the sender sends data packets and context information to the receiver. Second, intermediate nodes detect congestion. Third, hop-by-hop and centralized congestion avoidance is applied to mitigate incipient 
congestion. Fourth, the receiver determines the critical level of the packet using sender context information. Both critical level and network rate feedback is relayed to the sender. Fifth, the sender determines sending rate and appends the critical level to outgoing packets. Sixth, intermediate nodes perform packet prioritization.

\section{Critical Data Management}

Critical data prioritization based on context is crucial to allow the network to adapt to changing conditions. We use Dey's [5] definition of context: "Context is any information that can be used to characterize the situation of an entity."

We define criticality as a function of context, data content and role. For example, data from a first responder may be more critical than from a volunteer. Examples of context are the sender's activity, location, network condition, proximity of other team members, and battery level.

TRIAGE provides a framework that enables decision makers to define criticality through high-level policies. Context and role information is appended to the IP packet header by the sender, while content information is obtained by examining the packet payload.

\section{Inferring Congestion and Congestion Avoidance}

We use a queue occupancy-based method to infer congestion, with $25 \%$ of intermediate node buffer length as the threshold value. An exponentially weighted moving average tracks the occupied buffer length and sets a congestion bit in the outgoing packet header whenever the length exceeds the threshold value.

We take a two-step congestion avoidance approach upon inferring congestion to prevent congestion collapse, which would adversely affect critical packet delay. First, hop-byhop congestion avoidance is done by setting a congestion bit in the outgoing packet header. Upstream nodes closer to the sender overhear these packets and reduce their sending rates while setting a congestion bit in their own outgoing packets until it reaches the sender. Intermediate nodes also include rate feedback in their outgoing packets.

The receiver then uses the collective rate feedback to perform centralized congestion avoidance. By having a broader view of the network, the receiver determines packet priority through both the intermediate node feedback and context information in the packet headers using high-level policies. The critical packet priority and network rate feedback are relayed to the sender by piggybacking on TCP acknowledgment packets.

\section{E. Sender Rate Adaption and Packet Prioritization}

The sender uses both these values to determine an appropriate sending rate, which will be a function of network rate feedback and critical level information. The challenge is in determining a rate that takes into account network congestion and critical level, which change dynamically based on user context.

The sender appends the critical packet level obtained from the receiver to their outgoing packets. Intermediate nodes along the network path use the information in the packet headers to prioritize transit packets by giving preference to critical packets over non-critical packets.

\section{EVALUATION}

To evaluate the effectiveness of our system, we plan to use three metrics: one-way delay, throughput, and fairness. Intuitively, our TRIAGE framework should reduce critical packet delay and increase throughput while slightly increasing delay for non-critical packets. Fairness is also needed to ensure that our framework does not heavily penalize non-critical packet flows.

We are currently conducting simulations using the ns-3 network simulator. We plan to run an experimental evaluation with Android-based mobile devices in a simulated disaster response scenario in the DART [6] collapsed structure testbed at NASA Ames Research Center.

\section{CHALLENGES}

In this paper, we describe a framework that is a first step towards ensuring timely delivery of critical data in mobile ad hoc networks. We placed several constraints on our initial design, which must be addressed to produce a system capable of working in a more realistic environment.

In hastily formed networks, it is not practical to assume network feedback. Thus, an end-to-end solution to infer network rate is desirable to complement our current design. Another possible implication is that non-critical data will experience slightly longer delays. One approach to overcome this is by aggregating non-critical data.

Finally, we wish to validate that our approach can apply to real-world disaster response scenarios. We plan to leverage knowledge from researchers and first responders in recent relief efforts, such as in Haiti, Chile and San Bruno [7] to design and model realistic scenarios and constraints.

\section{ACKNOWLEDGMENT}

I would like to thank my advisor, Martin Griss, for his invaluable advice and support. I also thank Patrick Tague for his insightful suggestions in improving this work and the anonymous reviewers for their thoughtful comments. This research is supported by the Agong Scholarship.

\section{REFERENCES}

[1] K. Sundaresan, V. Anatharaman, H.Y. Hsieh, "ATP: A Reliable Transport Protocol for Ad-Hoc Networks" ACM MobiHoc 2003.

[2] B. Hull, K. Jamieson, H. Balakrishnan, "Mitigating Congestion in Wireless Sensor Networks", SenSys 2004

[3] M. Zorzi, R. Rao, "Geographic Random Forwarding (GeRaF) for Ad Hoc and Sensor Networks: Multihop Performance", IEEE Transactions on Mobile Computing, 2003.

[4] S.Y. Oh, D. Lau, M. Gerla, "Content Centric Networking in Tactical and Emergency MANETs", Wireless Days, IFIP 2010.

[5] A. K. Dey, G. D. Abowd, "Towards a Better Understanding of Context and Context-Awareness", CHI 2000.

[6] Disaster Assistance and Rescue Team, http://dart.arc.nasa.gov/.

[7] J. Stamberger, "San Bruno Fire Technical Debrief", Carnegie Mellon Silicon Valley Disaster Management Initiative, November 2010. 